\title{
Validade da Escala de Cognições Acadêmicas Autorreferentes: autoconceito, autoeficácia, autoestima e valor
}

\author{
Bianca Cristine Gomide Costa ${ }^{1}$, Cristiano Mauro Assis Gomes \\ Universidade Federal de Minas Gerais - Belo Horizonte-MG, Brasil \\ Denise de Souza Fleith \\ Universidade de Brasília - Brasília-DF, Brasil
}

\section{RESUMO}

Este estudo objetivou construir e investigar evidências de validade estrutural da Escala de Cognições Acadêmicas Autorreferentes, que avalia autoconceito, autoeficácia e autoestima escolar e valor atribuído à habilidade escolar. Participaram do estudo 812 estudantes do ensino médio de escolas públicas e privadas de Minas Gerais. Os dados foram analisados com modelamento por equação estrutural, análise fatorial confirmatória e teste de $\alpha$ de Cronbach. O modelo estrutural proposto apresentou bom ajuste aos dados: $\chi^{2}[145]=475,299$; índice de ajuste comparativo $(\mathrm{CFI})=0,98$; raiz quadrada média do erro de aproximação (RMSEA) $=0,053$. Todas as relações item-fator propostas apresentaram betas maiores do que 0,30. Foram encontrados índices razoáveis de consistência interna: autoconceito escolar, $\alpha=0,69$; valor atribuído à escola, $\alpha=0,79$; autoeficácia escolar, $\alpha=0,74$; e autoestima escolar, $\alpha=0,72$. Os dados fornecem respaldo empírico quanto à validade estrutural da escala. Esse instrumento representa um avanço nas medidas de self, apontando a possibilidade de uma definição e mensuração diferencial dos construtos.

Palavras-chave: validade do teste; validade estatística; psicometria; self.

ABSTRACT - Validity of the Self-Referential Cognitions Scale: Self-concept, self-efficacy, self-esteem and value The aim of this study was to develop and investigate the structural validity of the Academic Self-Referential Cognitions Scale, which assesses academic self-concept, self-efficacy, and self-esteem, as well as the value assigned to school skills. The sample consisted of 812 high school students from public and private schools from the state of Minas Gerais, Brazil. Confirmatory factor analysis, structural equation modeling, and Cronbach's alpha test were used to analyze the data. The structural model proposed presented good fit to data: $\chi^{2}[145]=475.299$; comparative fit index $(\mathrm{CFI})=0.98$; root mean square error approximation $(\mathrm{RMSEA})=0.053$. All of the itemfactor relations proposed showed beta values higher than 0.30 . The reliability indices were reasonable: school self-concept, $\alpha=0.69$; value assigned to school, $\alpha=0.79$; school self-efficacy, $\alpha=0.74$; school self-esteem, $\alpha=0.72$. The data provide empirical support to the structural validity of the scale. This instrument represents an advancement in the measures of self, indicating the possibility of a differential definition and measurement of the constructs.

Keywords: test validity; statistical validity; psychometrics; self.

RESUMEN - Validez de la Escala de Cogniciones Académicas Autoreferenciales: autoconcepto, autoeficácia, autoestima y valor

Este estudio tuvo como objetivo la construcción y la investigación de la validez estructural de la Escala de Cogniciones Académicas Autoreferenciales, que evalúa el autoconcepto, la autoeficacia y la autoestima académicas y el valor asignado a la habilidad escolar. Participaron del estudio 812 estudiantes de secundaria de escuelas públicas y privadas de Minas Gerais, Brasil. Los datos fueron analizados mediante análisis factorial confirmatorio, modelos de ecuaciones estructurales y test $\alpha$ de Cronbach. El modelo estructural propuesto mostró un buen ajuste a los datos: $\chi^{2}[145]=475,299$; índice de ajuste comparativo (CFI)=0,98; raíz del resíduo quadrático promedio de aproximación (RMSEA) $=0,053$. Todas las relaciones ítem-factor propuestas tuvieron betas superiores a 0,30 . Fueron encontrados razonable niveles de consistencia interna: autoconcepto, $\alpha=0,69$; valor, $\alpha=0,79$; autoeficacia escolar, $\alpha=0,74$; autoestima escolar, $\alpha=0,72$. Los datos proporcionan apoyo empírico a la validez estructural de la escala. Este instrumento representa un avance en las medidas de self, indicando la posibilidad de una definición y medición diferenciales de los constructos.

Palabras clave: validación de test; validación estadística; psicometría; self. 
A visão que o aluno tem de si mesmo é apontada como um importante fator para o desenvolvimento saudável e o desenvolvimento escolar (Bandura, 1977; Bracken, 2009; Trautwein \& Möller, 2016). Diversas pesquisas têm investigado o papel de variáveis ligadas ao self, em especial o autoconceito, a autoeficácia e a autoestima, no desenvolvimento do aluno, nos processos de ensino-aprendizagem e no rendimento acadêmico. Evidências indicam que crenças positivas de si mesmo podem atuar como fatores de proteção ao aluno no ambiente escolar, sendo capazes de mediar os efeitos negativos da ansiedade, da solidão e do perfeccionismo, reduzindo estresse, sintomas depressivos, burnout e favorecendo a satisfação com a vida dos estudantes (Galla \& Wood, 2011; Luo, Wang, Zhang, Chen, \& Quan, 2016; Tu \& Zhang, 2014). No que se refere ao desempenho escolar, os estudos têm constatado que o autoconceito, a autoestima e a autoeficácia possuem papel relevante na explicação e na predição do desempenho acadêmico (Parker, Marsh, Ciarrochi, Marshaal, \& Abduljabbar, 2013; Piran, 2014; Prince \& Nurius, 2014; Richardson, Abraham, \& Bond, 2012; Trautwein \& Möller, 2016). Além disso, pesquisas apontam relações de autoconceito, autoeficácia e autoestima com variáveis como dificuldades de aprendizagem (Guerreiro, 2015), motivação e engajamento (Guerreiro, 2015; Green et al., 2012), bem-estar e emoções (Goetz, Cronjaeger, Frenzel, Lüdtke, \& Hall, 2010; Tu \& Zhang, 2014), etc. Há concordância entre os autores citados de que uma visão positiva de si mesmo está associada a uma melhor trajetória escolar.

Buscando esclarecer como as crenças do aluno sobre si mesmo afetam sua trajetória acadêmica, Trautwein e Möller (2016) apontam a existência de uma relação entre o engajamento do aluno com uma dada área e a importância que ele atribui à mesma. Para os autores, o interesse é um dos determinantes do autoconceito acadêmico, existindo um forte vínculo entre o que o aluno acredita que pode fazer e o que ele gosta de fazer. Ou seja, para além da visão de si mesmo, a avaliação que o indivíduo faz das tarefas que lhes são apresentadas é apontada como mediadora entre a pessoa e o ambiente, definindo a adoção de estratégias de comportamento, esforço, motivação e persistência frente à tarefa (Folkman, 2013). Nessa direção, em um estudo realizado com 398.750 alunos de 15 anos de idade de 57 países, Nagengast et al. (2011) encontraram um robusto subsídio empírico de que o valor atribuído interage com o autoconceito na explicação do envolvimento com a tarefa. Diante das evidências de uma estreita associação entre a visão de si mesmo e o interesse do aluno (Trautwein \& Möller, 2016), o presente estudo, além de investigar o autoconceito, a autoeficácia e a autoestima escolar, investiga também o valor atribuído pelo aluno às habilidades escolares.

Buscando as origens dos estudos acerca do self, constata-se que uma das primeiras considerações teóricas sobre o termo foi apresentada por William James (1890/n.d.). James apresenta o self como um construto multifacetado, composto por um "eu-sujeito", conhecedor do mundo, e um "eu-objeto", objeto do próprio conhecimento. Para James, este último refere-se a tudo aquilo que o indivíduo reconhece como pertencente ao seu "eu" e pode apresentar importante papel na explicação e no entendimento do comportamento humano. Desde então, novas definições de self foram desenvolvidas, inclusive com o surgimento de outros conceitos derivados da noção que o indivíduo tem de si mesmo (ver Da Silveira, De Souza, \& Gomes, 2010; Demetriou, 2003; Epstein 1973; Giffard et al., 2013; Rogers, 1975). De forma geral, os construtos propostos como ligados ao self são apresentados como fenômenos complexos, dinâmicos, multidimensionais e fortemente influenciados pelas experiências sociais. No entanto, apesar dos pontos comuns, a infinidade de termos ligados ao self, com diferentes definições e a partir de abordagens diversas, traz à área problemas na definição conceitual de seus construtos, especialmente quanto à definição diferencial dos termos (Bong \& Skaalvik, 2003; Byrne, 1996).

No que se refere ao autoconceito, observa-se a existência de uma gama de diferentes propostas conceituais (Epstein 1973; Gecas, 1982; Marsh \& Shavelson, 1985; Rogers, 1975). Apesar de existir um consenso de que o autoconceito refere-se à percepção ou representação que o sujeito tem sobre si próprio (Neves \& Faria, 2009), por tratar-se de uma definição ampla, ainda há confusões teórico-conceituais na delimitação mais específica do termo. Simel (2013), por exemplo, apresenta esse construto como um sinônimo de autoimagem que, segundo a autora, seria composto por sentimentos sobre valor próprio e autoconfiança. Tal definição, no entanto, aproxima o autoconceito da autoestima e da autoeficácia. Já a autoeficácia, proposta por Bandura (1977), é definida como a convicção da própria pessoa de que ela pode executar com sucesso os comportamentos necessários para alcançar um resultado específico, sendo ligada não a uma expectativa de resultado, mas à confiança nas próprias capacidades. Apesar da autoeficácia possuir uma delimitação conceitual bem estabelecida, ainda há problemas quanto à sua diferenciação do autoconceito (Bong \& Skaalvik, 2003). A autoestima, por sua vez, pode ser definida como a soma das avaliações afetivas feitas pelo indivíduo acerca de seu próprio valor ou importância (Blascovich \& Tomaka, 1991). Esse construto é normalmente apresentado como uma parcela avaliativa do autoconceito (Blascovich \& Tomaka, 1991; Martin-Albo, Nunez, Navarra, \& Grijalvo, 2007), sendo defendido que a distinção entre o autoconceito e a autoestima estaria no fato de que a última envolve a valorização de si por meio de um referencial afetivo, enquanto o primeiro refere-se a uma autopercepção e julgamento de si mesmo, constituindo um referencial cognitivo mais global (Byrne, 1996). No entanto, Byrne (1996) aponta que as evidências sobre a validade de construto não têm sido bem-sucedidas em sustentar empiricamente tais diferenças.

A partir do exposto, observa-se a existência de definições complementares ou por vezes sobrepostas, diante das quais 
não fica bem estabelecido se autoconceito, autoeficácia e autoestima são construtos independentes ou se são fenômenos complementares, que juntos integram uma noção mais ampla de self. Tais questões conceituais, ainda não solucionadas, geram dois principais problemas ao estudo dos fenômenos:

1. a investigação isolada dos conceitos, caso eles sejam expressões complementares de um mesmo fenômeno, pode subestimar o impacto e a força dos construtos na predição de comportamentos e na compreensão de seus papéis no desenvolvimento humano; e

2. a ausência de definições conceituais claras desencadeia problemas na mensuração adequada dos fenômenos, especialmente no que se refere à elaboração de itens específicos de cada construto.

Quanto à relevância da mensuração conjunta dos fenômenos, corroboramos as proposições de Nagengast et al. (2011) e Trautwein e Moller (2016), de que as relações entre a visão do aluno sobre si mesmo e o interesse não se resumem a uma mera soma dos efeitos individuais de cada variável, mas há uma relação multiplicativa entre elas que, quando articuladas, se potencializam na explicação dos resultados acadêmicos. Fundamentados nas correlações significativas usualmente encontradas entre as variáveis ligadas ao self (Oliveira, 2016; Parker et al., 2013), bem como nas correlações entre essas variáveis e o valor (Nagengast et al., 2011; Trautwein \& Moller, 2016), defendemos que, ao serem estudadas conjuntamente, as variáveis aqui apresentadas podem apresentar um padrão de correlações e interações que ultrapasse o estudo dos efeitos individuais de cada construto.

No que se refere à questão da medida, observa-se no cenário nacional a utilização de uma série de instrumentos para a mensuração do autoconceito (Suehiro, Rueda, Oliveira, \& Pacanaro, 2009), da autoeficácia (Martinelli, Bartholomeu, Caliatto, \& Sassi, 2009; Polydoro \& Guerreiro-Casanova, 2010; Sbicigo, Teixeira, Dias \& Dell'Aglio, 2012; Souza \& Brito, 2008), e da autoestima (Avanci, Assis, Santos, \& Oliveira, 2007; Hutz \& Zanon, 2011; Sbicigo, Bandeira, \& Dell'Aglio, 2010). Trata-se, de maneira geral, de instrumentos de autorrelato, com afirmativas perante as quais o sujeito indica o quanto a afirmação feita reflete a visão que ele possui de si mesmo no aspecto descrito. A utilização de instrumentos diversos, que partem de delimitações conceituais diferentes para a avaliação de um mesmo construto, dificulta a comparabilidade dos dados, bem como a generalização dos resultados. Por existirem múltiplas definições de um mesmo fenômeno, os itens de cada instrumento irão enfocar aspectos diferentes, o que leva à mensuração de fenômenos distintos, mas identificados sob o mesmo rótulo. Além disso, itens que se propõem a avaliar construtos diferentes, por vezes, apresentam redações muito semelhantes, avaliando na realidade um mesmo fenômeno. Por exemplo, os três itens:

- "Eu sou capaz de fazer coisas tão bem quanto a maioria das pessoas";
- "Eu tenho mais dificuldades para aprender do que os meus colegas"; e,

- "Eu consigo falar bem na frente da classe"

referem-se a uma autoavaliação da própria habilidade em comparação aos outros, sendo possível inferir que medem em seus sujeitos um mesmo fenômeno. No entanto, o primeiro foi retirado de uma escala de autoestima (Avanci et al., 2007, p. 403), o segundo é referente à avaliação da autoeficácia (Medeiros, Loureiro, Linhares, \& Marturano, 2000, p. 105) e o terceiro se propõe a avaliar o autoconceito (Stevanato, Loureiro, Linhares, \& Marturano, 2003, p. 72). Destaca-se, ainda, que não foram encontrados na literatura instrumentos que propusessem a avaliação dos três construtos de maneira conjunta. Diante do exposto, o presente estudo teve como objetivo construir e investigar a validade estrutural e consistência interna da Escala de Cognições Acadêmicas Autorreferentes (ECAA), construída para medir o autoconceito, a autoeficácia e a autoestima escolar, bem como o valor atribuído à habilidade escolar.

\section{Método}

\section{Participantes}

A amostra do estudo foi composta de 812 estudantes do ensino médio, sendo 378 (46,55\%) do sexo masculino e 434 $(53,45 \%)$ do sexo feminino, provenientes de 3 escolas públicas estaduais e 1 escola privada da cidade de Belo Horizonte, Minas Gerais, e 1 escola pública federal e 1 escola privada da cidade de Viçosa, Minas Gerais. As escolas foram escolhidas por conveniência. A média de idade dos participantes foi de 16,5 anos, com desvio-padrão (DP) de 1,25 anos.

\section{Procedimentos de construção do instrumento}

Neste estudo, entende-se o autoconceito, a autoeficácia e a autoestima como construtos multidimensionais, hierárquicos, dinâmicos, desenvolvidos pelas experiências sociais e relevantes para o funcionamento adequado dos indivíduos. Baseando-se nessa concepção, optou-se pela construção de um instrumento no qual as cognições autorreferentes do aluno fossem avaliadas a partir de habilidades específicas ligadas ao contexto educativo. Após a revisão de literatura, definiu-se conceitualmente os construtos para embasar a ECAA. Autoconceito foi entendido como a visão que o sujeito tem de si mesmo, uma autodescrição ligada à sua identidade pessoal. Diante de uma situação ou habilidade escolar específica, o autoconceito acadêmico é definido como o senso de identidade que a pessoa tem em relação àquele aspecto específico. Valor foi definido como o grau de importância que a pessoa atribui a um determinado domínio ou habilidade escolar específica. Autoeficácia foi entendida com base na proposição clássica de Bandura (1977) e definida como o julgamento ou avaliação que a pessoa elabora sobre sua capacidade de realizar corretamente certas 
tarefas ou atividades de um domínio particular. No caso do instrumento, o quanto a pessoa se julga capaz frente ao domínio escolar. O construto autoestima também não apresenta definição consensual na literatura. Foi entendido como o julgamento emocional da pessoa sobre si mesma e o quanto ela se sente bem consigo em função de sua relação com determinado domínio ou habilidade específica (no caso do instrumento, o domínio escolar).

Realizada a definição de cada construto, foram elaborados itens que buscassem expressar adequadamente cada conceito. Os itens deveriam ser genéricos, de forma a serem capazes de contemplar diferentes habilidades escolares selecionadas. Os itens ficaram com a seguinte estrutura frasal: para avaliar o autoconceito escolar, "O quanto a habilidade X tem a ver com você?"; para aferir o valor atribuído à habilidade escolar, "O quanto você valoriza a habilidade X?"; para avaliar autoeficácia, "Como você costuma se sair em tarefas que envolvem a habilidade X?"; e para medir autoestima, "Em relação a sua habilidade X, como você se sente?". Os itens, juntamente com a definição de cada cognição autorreferente, foram apresentados a um juiz, psicólogo, doutor em Psicologia, com vasta experiência em psicometria, construção de testes psicológicos e testes psicométricos para educação. A partir das sugestões do juiz, foram feitas alterações visando uma melhor compreensão dos itens, bem como uma melhor representatividade dos conceitos. Posteriormente, selecionou-se um conjunto de 30 habilidades escolares específicas que permitissem avaliar tais conceitos de forma contextualizada. Essas habilidades foram expostas ao referido juiz, que teve a função de selecionar as dez habilidades mais pertinentes ao contexto escolar, que preenchessem os pré-requisitos de importância para o processo de aprendizagem, contemplação de habilidades essenciais à escola e que fossem relevantes a pelo menos três disciplinas escolares. Foram apresentadas sugestões e modificações na forma de descrição das habilidades. As habilidades selecionadas foram:
1. interpretação de textos;

2. escrita;

3. hábito de estudar;

4. atenção durante as aulas;

5. compreensão de novos conteúdos;

6. solução de equações matemáticas;

7. realização de contas de cabeça;

8. manutenção das tarefas em dia;

9. apresentação oral de trabalhos; e

10. realização de provas e avaliações.

Após o processo de construção e ajuste de itens, o instrumento passou por uma aplicação piloto, com 126 sujeitos, idade média de 28,37 anos $(D P=12,72)$, a fim de verificar a funcionalidade da escala quanto aos seguintes aspectos: aplicabilidade do instrumento, possíveis erros de digitação, análise semântica e impressões dos sujeitos ao instrumento. A partir dos resultados desse estudo piloto, foram feitas mudanças na redação do instrumento, chegando à versão final que será apresentada a seguir.

\section{Instrumento}

A ECAA (Costa, 2014; 2016) é um instrumento de autorrelato desenvolvido para mensurar o autoconceito escolar, a autoeficácia escolar, a autoestima escolar e o valor atribuído à habilidade escolar em alunos do ensino médio. $\mathrm{O}$ instrumento avalia cada um dos quatro construtos considerando as dez habilidades escolares mencionadas anteriormente. Diante de cada habilidade escolar, o aluno deve responder o quanto aquela habilidade tem a ver com ele (autoconceito), o quanto ele valoriza aquela atividade (valor), o quanto ele julga ser capaz de realizar tarefas que envolvem aquela habilidade (autoeficácia) e como ele se sente diante daquela habilidade específica (autoestima). O instrumento é composto por 40 itens no total, sendo dez por construto. A escala de resposta aos itens é de cinco pontos. A Figura 1 ilustra a composição

\section{A - Em relação à interpretação de textos:}

1 - O quanto a interpretação de textos tem a ver com você?

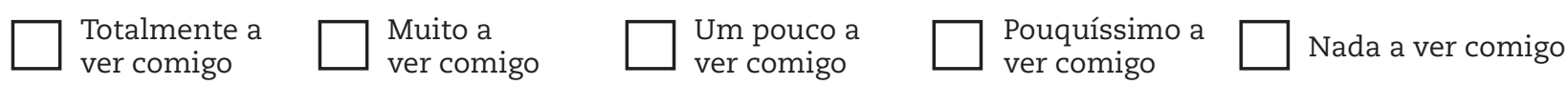

2 - O quanto você valoriza a interpretação de textos?
$\square$ Acho essencial
Acho importante
Valorizo
$\square$ Valorizo pouco
Não valorizo

3 - Como você costuma se sair em tarefas que envolvam a interpretação de textos?
$\square$ Muito bem
$\square$ Bem
$\square$ Mais ou menos
$\square \mathrm{Mal}$
Muito mal

4 - Em relação à sua capacidade de interpretação de textos, como você se sente?

Totalmente satisfeito

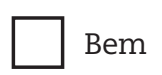

$\square$ Indiferente

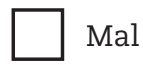

Um fracasso

Figura 1. Estrutura básica da Escala de Cognições Acadêmicas Autorreferentes 
básica da escala para uma das habilidades escolares. A estrutura se repete, modificando apenas a habilidade escolar em questão. A aplicação do instrumento pode ser tanto individual quanto coletiva e não há limite de tempo.

A Figura 2 é a representação gráfica do modelo fatorial proposto pela ECAA. Esse modelo sugere a mensuração de quatro variáveis latentes correlacionadas entre si: autoconceito (Ac), autoeficácia (Aef), autoestima (Aes) e valor atribuído à escola $(\mathrm{Va})$. Cada um desses fatores é composto por 10 itens: autoconceito (i1, i5, i9, i13, i17, i21, i25, i29, i33 e i37), valor atribuído à escola (i2, i6, i10, i14, i18, i22, i26, i30, i34 e i38), autoeficácia (i3, i7, i11, i15, i19, i23, i27, i31, i35 e i39) e autoestima (i4, i8, i12, i16, i20, i24, $\mathrm{i} 28$, i32, i36 e i40). Considerando que a cada quatro itens do instrumento há uma habilidade escolar diferente sendo enfocada, hipotetiza-se que o conjunto de quatro itens relacionados à mesma habilidade escolar de referência (um de Ac, um de Aef, um de Aes e um de Va) será explicado por um fator de autorreferrência da habilidade específica.
Essas dez variáveis latentes geradas por cada habilidade específica não se correlacionam com as quatro variáveis latentes de autoconceito, autoeficácia, autoestima e valor. $\mathrm{Na}$ Figura 2, esses fatores estão representados por $\mathrm{AH}$ e são ligados aos seus itens correspondentes.

\section{Procedimentos de coleta de dados}

O presente trabalho foi aprovado pelo Comitê de Ética em Pesquisa da Universidade Federal de Minas Gerais. Para a realização da pesquisa foram contatadas, no ano de 2013, 20 escolas públicas e privadas, nas 2 cidades investigadas. Das escolas convidadas, seis concordaram em fazer parte da pesquisa. A ECAA foi aplicada na própria sala de aula pela pesquisadora e estudantes de psicologia devidamente treinados, com auxílio dos professores das escolas. Previamente ao preenchimento do instrumento, foi realizada uma explicação do objetivo da pesquisa e distribuídos Termos de Consentimento Livre e Esclarecido aos alunos e pais.

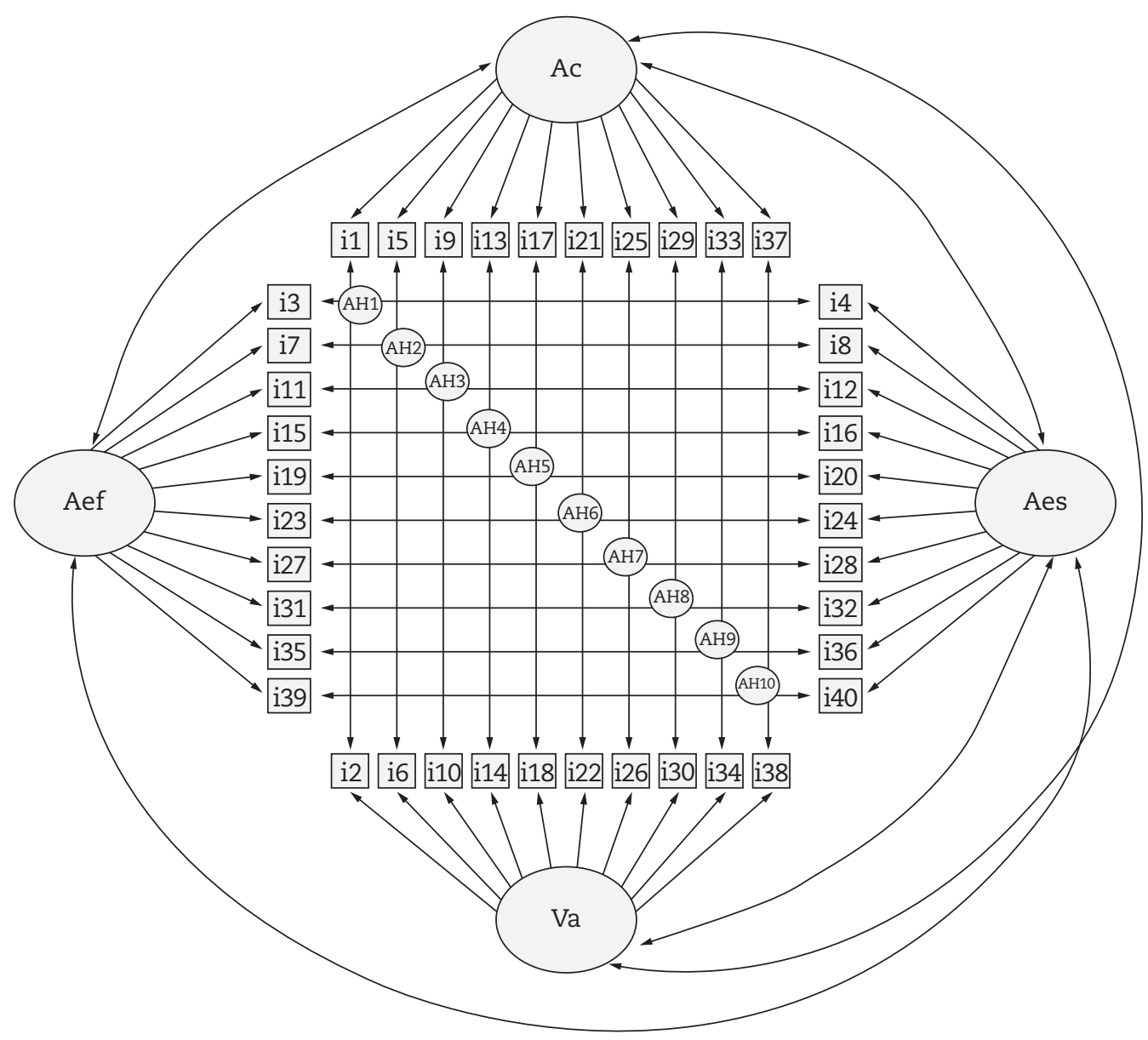

Nota: Ac=autoconceito escolar; Aef=autoeficácia escolar; Aes=autoestima escolar; $\mathrm{Va}=$ valor escolar; $\mathrm{AH}=$ autorreferência de habilidade específica.

Figura 2. Representação gráfica do Modelo Estrutural da Escala de Cognições Acadêmicas Autorreferentes 


\section{Procedimentos de análise de dados}

Para análise dos dados foram utilizadas as técnicas de análise fatorial confirmatória e modelamento por equação estrutural por meio do software estatístico Mplus 6.0. O método empregado para estimativa dos dados foi o dos quadrados mínimos ponderados robustos - robust weighted least squares (WLSMV) -, adequado para a estimativa de dados categóricos. O modelo teórico proposto para o instrumento foi testado quanto ao seu grau de ajuste aos dados e foi avaliado pelo índice de ajuste comparativo - comparative fit index (CFI) - e pela raiz quadrada média do erro de aproximação - root mean square error approximation (RMSEA). Um bom ajuste aos dados é indicado por valores do CFI iguais ou superiores a 0,95 ; e valores do RMSEA iguais ou inferiores a 0,06 (Blunch, 2008). Por fim, foram investigadas as confiabilidades das medidas de autoconceito, autoeficácia, autoestima e valor por meio do teste de $\alpha$ de Cronbach.

\section{Resultados}

Inicialmente, para a definição das estratégias de análise de dados adequadas à presente amostra, foi realizado um teste de normalidade, sendo encontrada uma distribuição multivariada não normal (curtose $=200,783$; valor de base $=49,352$; dados obtidos por meio do software estatístico AMOS 16.0). Posteriormente, foi realizado teste de adequação do modelo estrutural proposto, que apresentou bom ajuste aos dados: $\chi^{2}[145]=475,299 ; \quad C F I=0,98$; e RMSEA $=0,053$. A Figura 3 traz a solução estrutural, os betas e correlações encontradas no modelo. Quanto à relação item-fator, observa-se, na Figura 3, que todas as relações estabelecidas no modelo possuem betas maiores do que 0,30 , sugerindo que as relações propostas são relevantes. Os betas dos itens em seus respectivos fatores de cognições autorreferentes variam de moderados a fortes: autoconceito teve seus itens

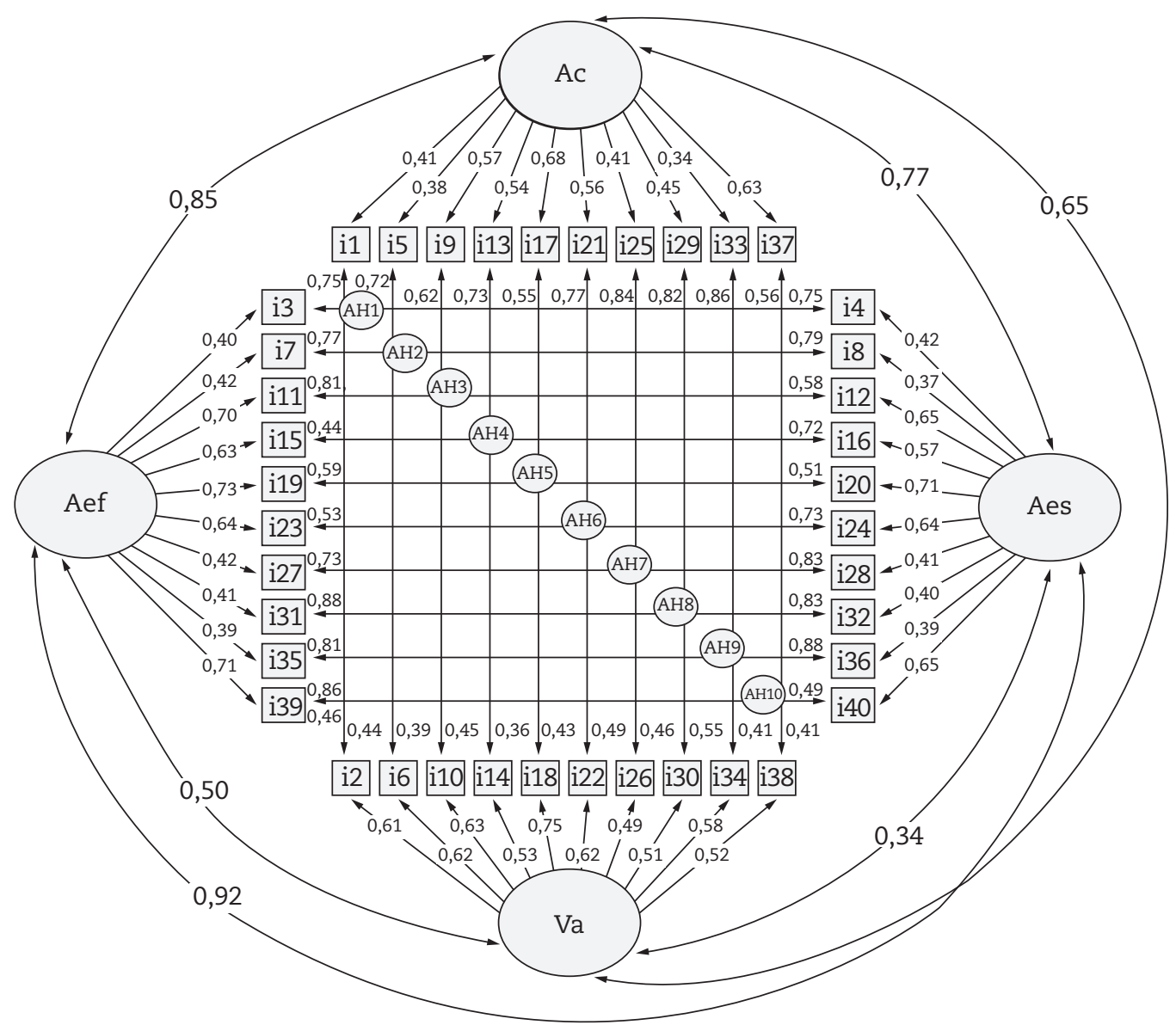

Nota: Ac=autoconceito escolar; Aef=autoeficácia escolar; Aes=autoestima escolar; Va=valor escolar; AH=autorreferência de habilidade específica; As correlações das autorreferências de habilidade específica entre si foram omitidas visando uma melhor visualização do modelo proposto.

Figura 3. Betas e correlações do modelo estrutural do teste 
com betas variando de 0,34 a 0,68 ; valor apresentou itens com betas de 0,49 a 0,75 ; os itens de autoeficácia tiveram betas que variavam de 0,39 a 0,73 ; e autoestima obteve betas variando de 0,37 a 0,71 . Os resultados apontam uma razoável confiabilidade das medidas: autoconceito escolar $(\alpha=0,69)$, valor escolar $(\alpha=0,79)$, autoeficácia escolar $(\alpha=0,74)$ e autoestima escolar $(\alpha=0,72)$. Ressalta-se que, segundo o modelo fatorial proposto, cada item é explicado por mais de um fator, uma vez que cada item faz referência tanto a uma cognição autorreferente quanto a uma habilidade escolar específica. A Figura 3 indica ainda que há, em cada item, significativa influência do seu contexto específico, uma vez que foram encontradas cargas moderadas a fortes entre os itens e seus fatores de habilidades escolares autorreferentes, com betas variando de 0,36 a 0,88 .

O modelo teórico da escala também propõe a existência de correlação entre os fatores de autoconceito, autoeficácia, autoestima e valor atribuídos à escola. Os resultados sustentam esse postulado, pois foram encontradas correlações moderadas a fortes (Tabela 1). Constata-se que os dados fornecem respaldo empírico quanto ao ajuste do modelo proposto teoricamente. A ECAA mostrou-se adequada para avaliar as quatro cognições autorreferentes propostas: autoconceito, valor, autoeficácia e autoestima, sendo cada um desses fatores composto por dez itens.

\section{Discussão}

Em 1996, Byrne apresentou uma importante revisão crítica do estado da arte em autoconceito, apontando limitações na definição conceitual do termo, na definição diferencial em relação à autoeficácia e à autoestima, e ainda problemas de mensuração desses construtos. Passados 20 anos dos apontamentos de Byrne, os problemas de definição diferencial dos construtos do self ainda persistem. Os resultados encontrados no presente estudo representam uma contribuição a essa problemática, indicando uma confirmação empírica da existência de autoconceito, autoestima, e autoeficácia como fatores autônomos e do mesmo nível. Isso significa dizer que os dados dão sustentação à hipótese de que é possível a identificação de

Tabela 1

Correlações entre Fatores de Cognição Acadêmica Autorreferentes

\begin{tabular}{lcccc}
\hline Fatores & 1 & 2 & 3 & 4 \\
\hline 1. Autoconceito & - & & & \\
2. Valor & $0,65^{*}$ & - & & \\
3. Autoestima & $0,77^{*}$ & $0,34^{*}$ & - & \\
4. Autoeficácia & $0,85^{*}$ & $0,50^{*}$ & $0,92^{*}$ & - \\
\hline Nota: ${ }^{*} p<0,05$. & & & &
\end{tabular}

três variáveis latentes diferentes quando tratamos da visão que o aluno tem de si mesmo: uma referindose a uma autoidentidade, outra a uma autoavaliação de capacidade e uma terceira referente a um autojulgamento de valor. Uma vez que o fator autoestima foi identificado no mesmo nível do autoconceito, os achados deste estudo, aparentemente, contrariam algumas proposições de que a autoestima poderia ser um subcomponente do autoconceito relativo a um autojulgamento de valor (Blascovich \& Tomaka, 1991; Martin-Albo et al., 2007).

De maneira semelhante, os resultados encontrados parecem indicar a não sustentação de algumas proposições teóricas sobre a diferenciação entre autoconceito e autoeficácia. Dois principais argumentos na literatura têm buscado diferenciar o autoconceito da autoeficácia: a especificidade e a influência do quadro de referência. Segundo a hipótese da especificidade, o autoconceito referir-se-ia a aspectos mais globais, ao tempo que a autoeficácia estaria ligada a domínios muito específicos (Bong \& Skaalvik, 2003; Byrne, 1996; Neves \& Faria, 2009; Scherer, 2013). Porém, segundo Marsh, Xu e Martin (2012), ambos os construtos têm provado serem multidimensionais, contendo tanto aspectos globais quanto domínios específicos, sendo essa distinção insustentável. Em concordância com Marsh et al. (2012) e Trautwein e Möller (2016), no presente estudo ambos os construtos foram propostos e identificados no nível específico referente às habilidades escolares, exemplificando que tanto o autoconceito quanto a autoeficácia podem ser mensurados no nível mais específico do domínio ligado à tarefa.

O outro ponto de distinção entre autoconceito e autoeficácia apresentado na literatura diz respeito ao efeito do quadro de referência (Bong \& Skaalvik, 2003; Byrne, 1996; Marsh et al., 2012; Neves \& Faria, 2009). É defendido que respostas a itens de autoconceito tenderiam a ser mais influenciadas por comparações sociais com os pares (referencial externo), ou por comparações de sua própria habilidade em outras tarefas (referencial interno). Em contrapartida, as respostas dos alunos para itens de autoeficácia tenderiam a ser julgamentos focados na capacidade em relação a uma tarefa muito específica e, assim, menos influenciados pelo quadro de referência social. No entanto, conforme apontam Marsh et al. (2012), essa questão referese mais a como os construtos são medidos do que a uma distinção dos conceitos em si, merecendo, pois, maior foco de investigação. No presente estudo, ambos os construtos foram mensurados e identificados a partir de um mesmo quadro de referência, no caso, as diferentes habilidades escolares específicas. Isso indica que tanto os itens de autoconceito quanto itens de autoeficácia podem ser relativos a referenciais externos, internos ou de tarefa, a depender da construção do instrumento. 
As definições teóricas apresentadas na presente escala e sustentadas empiricamente parecem apontar que a diferenciação dos construtos reside não em questões de dimensão, hierarquia, contexto ou quadro de referência, mas em diferentes aspectos de desenvolvimento do self, que são contemplados por cada um dos fatores. Em síntese, parecem coexistir diferentes tipos de visões de si mesmo, de mesmo nível e especificidade: autoidentidade, autojulgamento de capacidade e autoavaliação emocional, estando todos relacionados à atribuição de valor dada ao contexto. Tais definições podem apontar caminhos para uma melhor identificação específica de cada construto, auxiliando na mensuração e avaliação dos aspectos do self, no avanço das pesquisas da área, bem como em intervenções psicológicas, já que fornecem pontos de distinção acerca das diferentes visões que um indivíduo pode ter de si mesmo e sua relação com o valor atribuído.

Importante frisar que, de forma semelhante a outras investigações (Oliveira, 2016; Parker et al., 2013; Souza \& Brito, 2008), foram encontradas correlações significativas, de moderadas a fortes, entre os construtos do self, indicando que, apesar de identificados como fatores autônomos, eles não são independentes um do outro. Essas correlações fortalecem a pertinência de se estudar esses fatores de forma associada. Uma vez que eles possuem importantes correlações entre si, o seu estudo coligado pode trazer uma melhor compreensão acerca do fenômeno do self. A critério de exemplo, o construto do valor atribuído, variável pouco comum nos estudos acerca da visão de si mesmo, se correlacionou em 0,$34 ; 0,50$ e 0,65 com autoestima, autoeficácia e autoconceito, respectivamente, corroborando a proposta de Nagengast et al. (2011) e Trautwein e Möller (2016) de que a importância que a pessoa dá ao domínio envolvido pode influenciar a visão que ela tem de si mesma ou vice-versa. Já a correlação de 0,92 encontrada entre autoestima e autoeficácia sugere a necessidade de maiores investigações acerca da identidade desses construtos. Pesquisas que proponham novos modelos de relação entre as variáveis podem agregar às discussões das definições diferenciais dos construtos.

Ressalta-se que a amostra do presente estudo não é representativa da população de estudantes do ensino médio brasileiro, sendo, portanto, necessárias novas investigações de validade estrutural e de invariância em outras amostras. Além disso, o estudo não abarcou a normatização da escala, sendo esse um apontamento futuro nos estudos da ECAA. Independentemente da necessidade de maiores avanços na investigação das diferenciações entre os conceitos, a ECAA apresentou bons indícios de validade estrutural, apresentando-se como um instrumento adequado a pesquisas e intervenções que visem avaliar autoconceito, autoeficácia e autoestima escolar, bem como valor atribuído à escola, em estudantes do ensino médio. Destacam-se como benefícios da escala a sua fácil aplicação, a mensuração conjunta de quatro variáveis diferentes relativas ao self, bem como a possibilidade de adaptação da mesma a outros contextos para além da escola.

\section{Agradecimentos}

Pesquisa financiada pelo Conselho Nacional de Desenvolvimento Científico e Tecnológico (CNPq) por meio de bolsa de mestrado concedida à primeira autora.

\section{Referências}

Avanci, J. Q., Assis, S. G., Santos, N. C., \& Oliveira, R. V. (2007). Adaptação transcultural da escala de autoestima para adolescentes. Psicologia: Reflexão e Crítica, 20(3), 397-405. Recuperado de http://www.scielo.br/pdf/prc/v20n3/a07v20n3.pdf

Bandura, A. (1977). Self-Efficacy: Toward a unifying theory of behavioral change. Psychological Review, 34(2), 191-215. Recuperado de https:// www.uky.edu/ eushe2/Bandura/Bandura1977PR.pdf

Blascovich, J., \& Tomaka, J. (1991). Measures of self-esteem. Em J. R. Robinson, R. Shaver \& L. S. Wrightman (Eds.), Measures of personality and social psychological attitudes (pp. 115-160). New York: Elsevier.

Blunch, N. J. (2008). Introduction to structural equation modeling using SPSS and AMOS. Thousand Oaks, CA: Sage.

Bong, M., \& Skaalvik, E. M. (2003). Academic self-concept and self-efficacy: How different are they really? Educational Psychology Review, 15(1), 1-40. Recuperado de http://www.jstor.org/stable/23361533

Bracken, B. A. (2009). Positive self-concepts. Em R. Gilman, E. S. Huebner \& M. J. Furlong (Eds), Handbook of positive psychology in school (pp. 89-106). New York, NY: Routledge.

Byrne, B. M. (1996). Measuring self-concept across the life span: Issues and instrumentation. Washington, DC: American Psychological Association.

Costa, B. C. G. (2014). Investigando a relação entre autoconceito, autoeficácia e autoestima: construção de evidências a partir da escala de cognições acadêmicas autorreferentes. Dissertação de Mestrado não publicada, Universidade Federal de Minas Gerais, Belo Horizonte, MG.

Costa, B. C. G. (2016). Escala de Cognições Acadêmicas Autorreferentes. Escola Positiva. Recuperado de http://psicologabiancacosta.wixsite. com/escolapositiva/instrumentos

Da Silveira, A. C., De Souza, M. L., \& Gomes, W. B. (2010). "Falar com seus botões": pelos meandros teóricos e empíricos das relações entre conversa interna, reflexividade e self. Estudos de Psicologia, 15(3), 223-231. Recuperado de http://www.scielo.br/pdf/epsic/v15n3/a01v15n3.pdf 
Demetriou, A. (2003). Mind, self and personality: Dynamics interactions from late childhood to early adulthood. Journal of Adult Development, 10(3), 151-171. doi: 10.1023/A:1023462229730

Epstein, S. (1973). The self-concept revisited: Or a theory of a theory. American Psychologist, 28(5), 404-416. doi: 10.1037/h0034679

Folkman, S. (2013). Stress: Appraisal and coping. Em M. D. Gellman \& J. R. Turner (Eds.), Encyclopedia of behavioral medicine (pp. 1913-1915). New York: Springer.

Galla, B. M., \& Wood, J. J. (2012). Emotional self-efficacy moderates anxiety-related impairments in math performance in elementary school-age youth. Personality and Individual Differences, 52, 118-122.

Gecas, V. (1982). The self-concept. Annual Review of Sociology, 8(1), 1-33. doi: 10.1146/annurev.so.08.080182.000245

Giffard, B., Viard, A., Dayan, J., Morel, N., Joly, F., \& Eustache, F. (2013). Autobiographical memory, self, and stress-related psychiatric disorders: Which implications in cancer patients? Neuropsychology Review, 23(2), 157-168. doi: 10.1007/ s11065-013-9233-6

Goetz, T., Cronjaeger, H., Frenzel, A. C., Lüdtke, O., \& Hall, N. C. (2010). Academic self-concept and emotion relations: Domain specificity and age effects. Contemporary Educational Psychology, 35, 44-58. doi: 10.1016/j.cedpsych.2009.10.001

Green, J., Liem, G. A. D., Martin, A. J., Colmar, S., Marsh, H. W., \& McInerney, D. (2012). Academic motivation, self-concept, engagement, and performance in high school: Key processes from a longitudinal perspective. Journal of Adolescence, 35, 1111-1122. doi: 10.1016/j. adolescence.2012.02.016

Guerreiro, A. M. A. (2015). O que a escola escreve dentro de mim: a disortografia e o seu impacte no autoconceito e na motivação escolar (Dissertação de mestrado). Universidade Fernando Pessoa, Porto, Portugal. Recuperado de: http://bdigital.ufp.pt/bitstream/10284/4708/1/Tese\%20 de\%20Mestrado_Adriana\%20Guerreiro.pdf

Hutz, C. S., \& Zanon, C. (2011). Revisão da adaptação, validação e normatização da Escala de Autoestima de Rosenberg. Avaliação Psicológica, 10(1), 41-49. Recuperado de http://pepsic.bvsalud.org/scielo.php?script=sci_arttext\&pid=S1677-04712011000100005

James, W. (n.d.). The principles of psychology. Em C. D. Green (Ed.), Classics in the history of psychology. Recuperado de http://psychclassics. yorku.ca/James/Principles/index.htm (obra original publicada em 1890).

Luo, Y., Wang, Z., Zhang, H., Chen, A., \& Quan, S. (2016). The effect of perfectionism on school burnout among adolescence: The mediator of self-esteem and coping style. Personality and Individual Differences, 88, 202-208. doi: 10.1016/j.paid.2015.08.056

Marsh, W. H., \& Shavelson, R. (1985). Self-concept: Its multifaceted hierarquical structure. Educational Psychologists, 20(3), 107-123. doi: 10.1207/ s15326985ep2003_1

Marsh, H. W., Xu, M., \& Martin, A. J. (2012). Self-concept: A synergy of theory, method, and application. Em K. R. Harris, S. Graham \& T. Urdan (Eds.). APA educational psychology handbook, Vol. 1: Theories, constructs, and critical issues (pp. 427-458). Washington, DC: American Psychological Association.

Martin-Albo, J., Nunez, J. L., Navarra, J. G., \& Grijalvo, F. (2007). The Rosenberg Self-Esteem Scale: Translation and validation in university students. The Spanish Journal of Psychology, 10(2), 458-467. Recuperado de https://www.ncbi.nlm.nih.gov/pubmed/17992972

Martinelli, S. C., Bartholomeu, D., Caliatto, S. G., \& Sassi, A. G. (2009). Children's Self-efficacy Scale: Initial psychometric studies. Journal of Psychoeducational Assessment, 27(2), 145-156. doi: 10.1177/0734282908325551

Medeiros, P. C., Loureiro, S. R., Linhares. M. B. M., \& Marturano, E. M. A. (2000). Autoeficácia e os aspectos comportamentais de crianças com dificuldade de aprendizagem. Psicologia: Reflexão e Crítica, 13(3), 327-336. doi: 10.1590/S0102-79722000000300002

Nagengast, B., Marsh, H. W., Scalas, L. F., Xu, M., Hau, K. T., \& Trautwein, U. (2011). Who took the "X" out of expectancy-value theory? A psychological mystery, a substantive-methodological synergy, and a cross-national generalization. Psychological Science, 22, $1058-1066$. doi: 10.1177/0956797611415540

Neves, S. P., \& Faria, L. (2009). Auto-conceito e auto-eficácia: semelhanças, diferenças inter-relação e influência no rendimento escolar. Revista da Faculdade de Ciências Humanas e Sociais, 6(1), 206-218. Recuperado de http://bdigital.ufp.pt/bitstream/10284/1330/2/206-218_\%20 FCHS06-5.pdf

Oliveira, A. K. C. (2016). Autoconceito, autoeficácia e parentalidade: crianças com deficiência física, com desenvolvimento típico e seus familiares (Tese de Doutorado). Universidade Federal de São Carlos, São Carlos. Recuperado de https://repositorio.ufscar.br/handle/ ufscar/7443

Parker, P. D., Marsh, H. W., Ciarrochi, J., Marshaal, S., \& Abduljabbar, A. S. (2013). Juxtaposing math self-efficacy and self-concept as predictors of long-term achievement outcomes. Educational Psychology, 34(1), 29-48. doi: 10.1080/01443410.2013.797339

Piran, N. A. (2014). The relationship between self-concept, self-efficacy, self-esteem and reading comprehension achievement: Evidence from Iranian EFL learners. International Journal of Social Science E Education, 5(1), 58-66. Recuperado de http://ijsse.com/sites/default/ files/issues/2014/v4-i5-2014/Paper-7.pdf

Polydoro, S. A. J., \& Guerreiro-Casanova, D. C. (2010). Escala de autoeficácia na formação superior: construção e estudo de validação. Avaliação Psicológica, 9(2), 267-278. Recuperado de http://www.redalyc.org/pdf/3350/335027283011.pdf

Prince, D. \& Nurius, P. S. (2014) The role of positive academic self-concept in promoting school success. Children and Youth Services Review, 43, 145-152. doi: 10.1016/j.childyouth.2014.05.

Richardson, M., Abraham, C. \& Bond, R. (2012). Psychological correlates of university students' academic performance: A systematic review and meta-analysis. Psychological Bulletin, 138(2), 353-387. doi: 10.1037/a0026838.

Rogers, C. R. (1975). A terapia centrada no paciente. São Paulo: Martins Fonte.

Sbicigo, J. B., Bandeira, D. R., \& Dell'Aglio, D. D. (2010). Escala de Autoestima de Rosenberg (EAR): validade fatorial e consistência interna. Psico, 15(3), 395-403.

Sbicigo, J. B., Teixeira, M. A. P., Dias, A. C. G., \& Dell'Aglio, D. D. (2012). Propriedades psicométricas da Escala de Autoeficácia Geral Percebida (EAGP). Psico, 43(2), 139-146. Recuperado de https://dialnet.unirioja.es/descarga/articulo/5163218.pdf

Scherer, R. (2013). Further evidence on the structural relationship between academic self-concept and self-efficacy: On the effects of domain specificity. Learning and Individual Differences, 28(1), 9-19. doi: 10.1016/j.lindif.2013.09.008

Simel, S. (2013). Education for a positive self-image in a contemporary school. Journal of Education Culture and Society, 2, 108-115. doi: 10.15503/jecs20132-108-115

Souza, L. F. N. I., \& Brito, M. R. F. (2008). Crenças de autoeficácia, autoconceito e desempenho em matemática. Estudos de Psicologia, 25(2), 193-201. Recuperado de http://www.scielo.br/pdf/estpsi/v25n2/a04v25n2.pdf 
Stevanato, I. S., Loureiro, S. R., Linhares, M. B. M., \& Marturano, E. M. (2003). Autoconceito de crianças com dificuldades de aprendizagem e problemas de comportamento. Psicologia em Estudo, 8(1) 67-76. Recuperado de http://www.scielo.br/pdf/pe/v8n1/v8n1a09.pdf

Suehiro, A. C. B., Rueda, F. J. M., Oliveira, E. Z., \& Pacanaro, S. V. (2009). Avaliação do autoconceito no contexto escolar: análise das publicações em periódicos brasileiros. Psicologia Ciência e Profissão, 29(1), 18-29. Recuperado de http://www.redalyc.org/html/2820/282021771003/

Trautwein, U., \& Möller, J. (2016). Self-concept: Determinants and consequences of academic self-concept in school contexts. Em A. A. Lipnevich, F. Preckel \& R. D. Roberts (Eds.), Psychosocial skills and school systems in the twenty-first century: Theory, research, and practice (pp 187-214). New York, NY: Springer.

Tu, Y., \& Zhang, S. (2014). Loneliness and subjective well-being among Chinese undergraduates: The mediating role of self-efficacy. Social Indicators Research, 124(3), 963-980. doi: 10.1007/s11205-014-0809-1

\section{Sobre os autores}

Bianca Cristine Gomide Costa é Doutoranda em Psicologia pela Universidade de Brasília (UnB). Bolsista de Doutorado pelo Conselho Nacional de Desenvolvimento Científico e Tecnológico (CNPq). À época da realização da pesquisa encontrava-se vinculada à Universidade Federal de Minas Gerais (UFMG), como aluna de mestrado, onde adquiriu seu título de Mestre em Psicologia. Psicóloga e Bacharel em Psicologia pela Universidade Federal de Juiz de Fora (UFJF).

Cristiano Mauro Assis Gomes é Psicólogo, Doutor em Educação. Coordenador do Laboratório de Investigação da Arquitetura Cognitiva (LAICO), da Universidade Federal de Minas Gerais. Professor do Departamento de Psicologia, do Programa de Pós-graduação em Psicologia e do Programa de Pós-graduação em Neurociências da Universidade Federal de Minas Gerais. Experiência em Psicologia Escolar e do Desenvolvimento. Bolsista de Produtividade do CNPq, nível 2. http://lattes.cnpq.br/2458326202205195

Denise de Souza Fleith é Psicóloga e Ph.D. pela University of Connecticut. É professora associada do Instituto de Psicologia da Universidade de Brasília e bolsista de produtividade 1 do CNPq. 